\title{
Martian climatic events on timescales of centuries: Evidence from feature morphology in the residual south polar ice cap
}

\author{
Shane Byrne and Andrew P. Ingersoll \\ Division of Geological and Planetary Sciences, California Institute of Technology, Pasadena, California, USA \\ Received 23 April 2003; accepted 5 June 2003; published 9 July 2003.
}

[1] Flat-floored, quasi-circular depressions on the southern residual cap of Mars have been observed to expand rapidly. Analysis of the size distribution combined with modeling of the growth process yields information about the ages of these features. We report on properties of a population of these features within a selected study area. We find a narrow size distribution that indicates a common formation time and a finite period during which new features were forming. Some change in environmental conditions occurred during this period, which we date at several Martian decades to centuries ago depending on modeled growth rates. We report on THEMIS data that reveals the nature of larger features outside our study area. Underlying water ice is exposed on the flat floors of these depressions near the outer walls. These features may record more than one growth phase, indicating that conditions conducive to growth may switch on and off with characteristic timescales of Martian centuries. INDEX TERMS: 5407 Planetology: Solid Surface Planets: Atmospheres-evolution; 5462 Planetology: Solid Surface Planets: Polar regions; 5464 Planetology: Solid Surface Planets: Remote sensing; 5470 Planetology: Solid Surface Planets: Surface materials and properties; 6225 Planetology: Solar System Objects: Mars. Citation: Byrne, S., and A. P. Ingersoll, Martian climatic events on timescales of centuries: Evidence from feature morphology in the residual south polar ice cap, Geophys. Res. Lett., 30(13), 1696, doi:10.1029/2003GL017597, 2003.

\section{Introduction}

[2] It has been known for decades [Leighton and Murray, 1966; Kieffer, 1979; Thomas et al., 1992] that the southern polar cap of Mars is overlain by a residual deposit of $\mathrm{CO}_{2}$ ice, that does not sublime during the summer season. More recent observations conducted with the Mars Orbiter Camera (MOC) have revealed this residual $\mathrm{CO}_{2}$ deposit to contain a wide range of landforms [Thomas et al., 2000]. Among the most interesting and populous are flat-floored quasi-circular pits with steep walls (Figure 1), that have been dubbed Swiss-cheese features (SCF's). No changes in shape or size were observed over timescales of a single season [Malin and Edgett, 2001; Thomas et al., 2000] but Malin et al. [2001] observed, using images separated by one Martian year, that the walls of these depressions are expanding laterally at rates of $1-3 \mathrm{~m} / \mathrm{yr}$. The rapidity of this expansion is only possible in a medium as volatile as $\mathrm{CO}_{2}$ ice. Byrne and Ingersoll [2003] (hereafter referred to as paper 1) modeled the evolution and growth of these depressions as a hole in a layer of $\mathrm{CO}_{2}$ ice underlain by

Copyright 2003 by the American Geophysical Union. 0094-8276/03/2003GL017597 water ice and matched their observed expansion rates and morphologic properties, including the flat floors and steep walls. This paper continues from paper 1 and has two goals.

[3] Firstly, we detail the size distribution of a subset of the total SCF population. We interpret this size distribution in terms of ages using observed and modeled growth rates. Secondly, we report on additional Thermal Emission Spectrometer (THEMIS) data [Christensen et al., 2003] that reveals the nature of a separate, more geometrically complicated, class of SCF and speculate on their relationship to the measured population.

[4] The features are briefly described in Section 2. Section 3 describes the observations and growth model used. Section 4 discusses possible ages for the population based on the facts presented in Section 3. The nature of the second class of SCF and its connection with the population originally mapped are discussed in Section 5. Implications for recent polar history are discussed in Section 6 .

\section{Feature Description}

[5] There are two types of SCF being discussed in this paper; archetypes of both are illustrated in Figure 1. Many of the following characteristics were noted by Thomas et al. [2000].

[6] The first type (see Figure 1a) is common within our study area (see Figure 2). They are a few hundred meters across and have a small spread in size range. All shadow measurements to date indicate a consistent depth of $8-10 \mathrm{~m}$. They vary from heart shaped to almost circular, with a clear symmetry axis that is aligned north-south (see Figure 3). This combination of characteristics has not been observed in SCF's elsewhere on the residual cap.

[7] The second type (see Figure 1b) occurs more widely on the residual cap and is not confined to any particular region. The defining characteristic of this type of SCF is an interior moat running around the inside of their walls with the central floor being slightly elevated (by about two meters). They tend to be larger, with diameters of up to $1-2 \mathrm{~km}$. The walls are generally less steep; the equatorward facing walls tend to be steeper than poleward facing walls. Depth measurements are possible from the Mars Orbiter Laser altimeter (MOLA) and are again consistently $8-10 \mathrm{~m}$.

[8] Where the walls are shallowly sloping it can be seen that the medium in which the SCF's are embedded is composed of several layers (see Figure 1b). SCF's change their appearance toward the latter part of the summer as the seasonal frost disappears. Their walls and moats appear to darken considerably relative to the surrounding terrain [Malin and Edgett, 2001]. The surrounding flat upper 


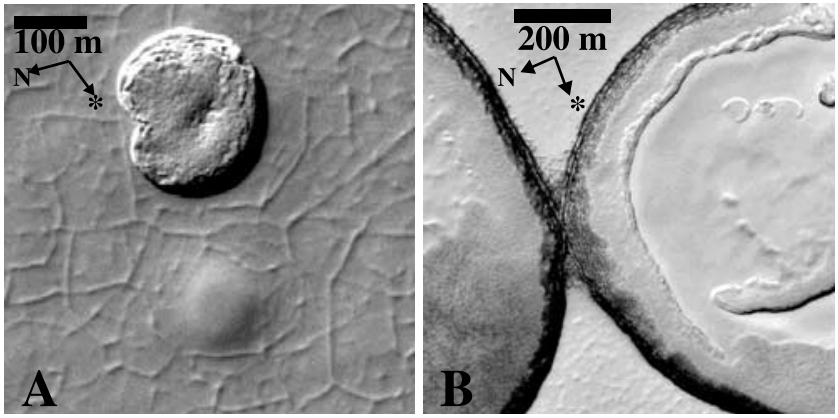

Figure 1. Examples of two different classes of SCF. In this and subsequent figures arrows denote the direction to the Sun $(*)$ and north (N). (a) Typical depression within our study area. Just below and to the right is a shallow bowl, which may be the progenitor of a future SCF. The grooved appearance of the surrounding terrain is commonly observed near these features in this area. Sub-frame of MOC narrow angle image M09/00609, taken at $87^{\circ} \mathrm{S}$, $353^{\circ} \mathrm{E}$, and $\mathrm{L}_{\mathrm{s}} 237^{\circ}$. (b) SCF showing a moat and raised central island. Also visible here is layering within the walls. Sub-frame of MOC narrow angle image M12/01995, taken at $86.9^{\circ} \mathrm{S}, 17^{\circ} \mathrm{E}$, and $\mathrm{L}_{\mathrm{s}} 305^{\circ}$.

surfaces and raised central islands do not to change in this way.

\section{Model and Observations}

[9] We choose a study region (indicated on Figure 2) of $400 \mathrm{~km}^{2}$ located close to the highest elevation on the residual cap. In many areas of the residual cap the SCF's have entirely coalesced and removed all the intervening flat

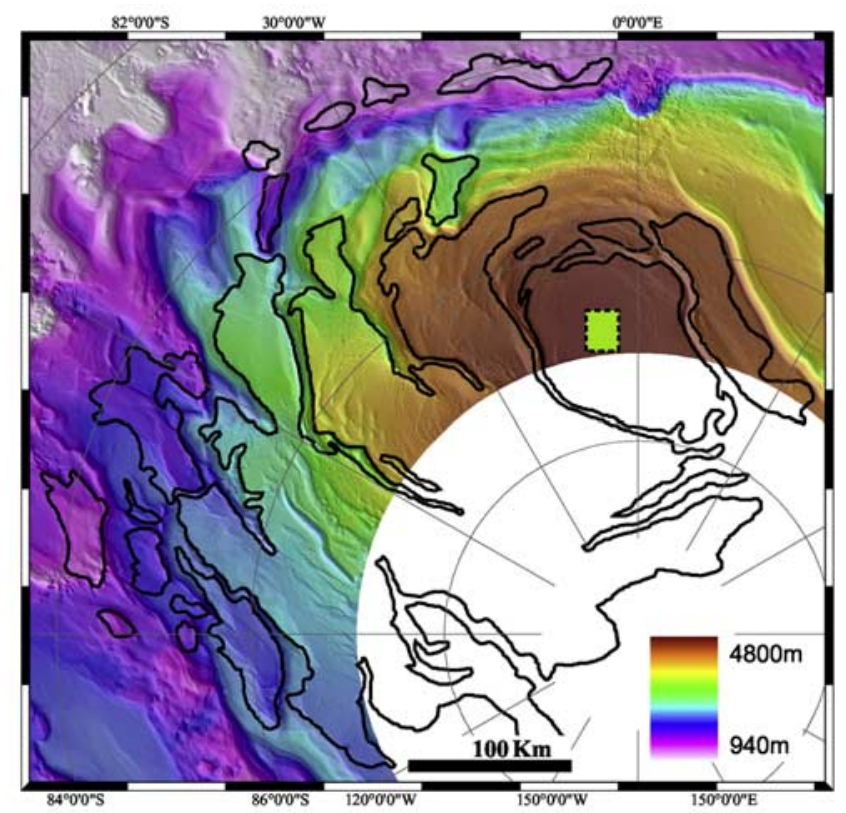

Figure 2. Shaded topographic map from MOLA of the southern residual cap (outlined in black [Kolb et al., 2003]). Data poleward of $87^{\circ} \mathrm{S}$ (white areas) is very sparse and not shown here. The region of interest discussed in this paper is outlined by the dashed square near $87^{\circ} \mathrm{S} 355^{\circ} \mathrm{E}$.

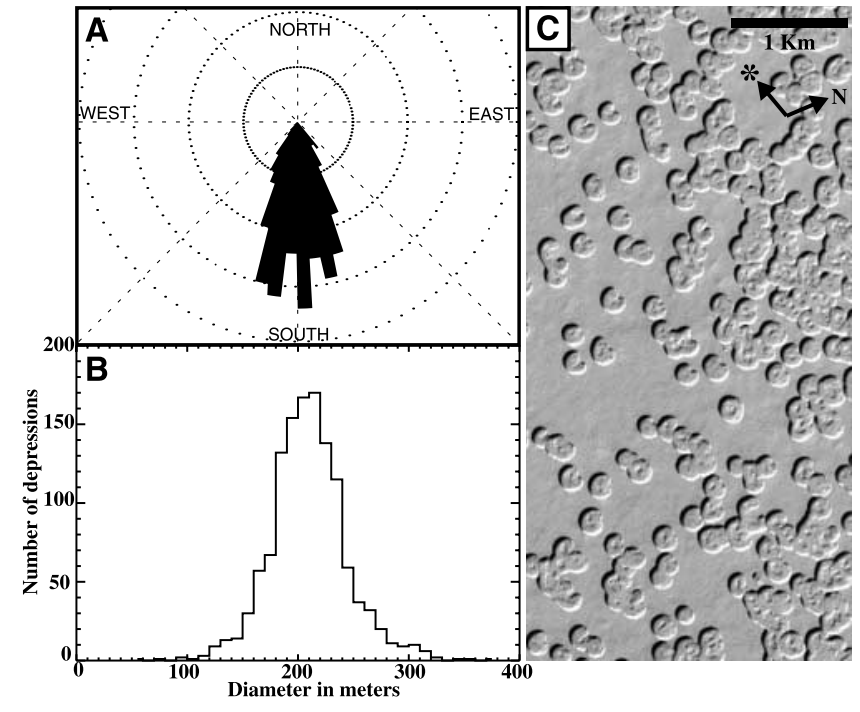

Figure 3. (a) Rose diagram of SCF orientations. The total number of features measured was 370 , the mean azimuth from the cusp to the center was within $0.2^{\circ}$ of south and the standard deviation was $\sim 17^{\circ}$. Concentric circles indicate number in increments of 10 . (b) Histogram of diameters of identifiable SCF's. The total number of features measured is 1263, the mean size was $217 \mathrm{~m}$ and the standard deviation was $\sim 35 \mathrm{~m}$. (c) Many SCF's destroying the upper $8 \mathrm{~m}$ thick layer in a sample view of our study area. Sub-frame of MOC narrow angle image M07/04167, taken at $86.8^{\circ} \mathrm{S}$, $355^{\circ} \mathrm{E}$, and $\mathrm{L}_{\mathrm{s}} 211^{\circ}$.

upper surfaces. In contrast, the SCF's located in our study area have areas of undisturbed surface between depressions so their sizes can be measured. In this regard this subset of the total SCF population is unique. The SCF expansion rates reported by Malin et al. [2001] relate to features observed outside of this study region where the $\mathrm{CO}_{2}$ ice layer is thinner. However, our modeling (see paper 1) indicates that the thickness of the $\mathrm{CO}_{2}$ ice layer has a negligible effect upon expansion rates.

[10] We combined 17 MOC narrow angle images together to cover $\sim 375 \mathrm{~km}^{2}$ ( $\sim 94 \%$ of the region outlined in Figure 2). The resolution of these frames ranged from 1.4 to 12 meters per pixel. SCF's were measured by marking line segments on the images to represent their diameters or, if significantly non-circular, their longest axis. Only features with greater than $270^{\circ}$ of intact azimuth were measured. Groups of features that had merged into amorphous shapes were not recorded. Center position and effective diameter were extracted from the line segments for analysis. Figure $3 \mathrm{c}$ shows a typical scene within our study area containing both isolated and merged depressions. We also measured the preferred pointing direction by drawing line segments from the cusp on the poleward facing wall (see Figure 1a) through the center of the depression. In general this was harder to measure because the cusps are only visible in the higher resolution images and many depressions are too circular to confidently identify the cusp position. For these reasons the number of features that have measured azimuths is much less than the number that have measured sizes.

[11] We measured the azimuths of 370 features within the study area (see Figure 3a). As can be seen qualitatively in 
Figure $3 \mathrm{c}$ the cusps are predominantly on the poleward facing walls. The mean orientation is almost exactly south $\left(0.17^{\circ}\right.$ off $)$ and the standard deviation is $\sim 17^{\circ}$. The northsouth alignment of the symmetry axis of these features argues strongly for an evolution connected directly to insolation. As we are not exactly at the pole the solar elevation decreases while the Sun is in the south. South-facing slopes therefore receive less sunlight and lose less mass than other walls of the depression. As the walls expand outward, the material on the south-facing wall is left behind and forms a cusp.

[12] In total we measured the sizes of 1263 features, which we identified as SCF's. The histogram of sizes is sharply peaked (see Figure 3b) with a mean diameter of $217 \mathrm{~m}$ and standard deviation of $35 \mathrm{~m}$. The narrow nature of the size distribution can also be seen qualitatively in Figure $3 \mathrm{c}$.

[13] We have developed a model to analyze the growth and evolution of SCF's with simple shapes [See paper 1]. We use growth rates from this model to interpret the measured size distribution. The model computes all orders of solar and re-emitted thermal radiation scattered within the depression. The depression is embedded within $\mathrm{CO}_{2}$ ice that is underlain by water ice. The model assumes cylindrical symmetry and all points on the inner surface of the depression must be visible from all other points (i.e., the shape of the depression must be concave at all locations), convex 'lumps' will mask some areas from others and so lead to inaccurate results. Within the confines of the above limit the depression can have an arbitrary shape that changes after each time step. Each part of the $\mathrm{CO}_{2}$ ice wall retreats/advances in response to its radiant energy surplus/ deficit. Exposed water ice can change temperature and conduct heat into and out of the subsurface.

\section{Age Relations}

[14] Our modeling indicates that the expansion of SCF's (after a short initial ramp-up phase) is linear in time. Wall retreat rates ranging from $0.5-2.5 \mathrm{~m} / \mathrm{yr}$ are possible with different subsurface albedo conditions (see paper 1). The sensitivity of the expansion rate to the albedo of the subsurface material introduces a large degree of uncertainty in trying to date these features. Differing concentrations of dust and other impurities within the $\mathrm{CO}_{2}$ ice from area to area could result in differing expansion rates so SCF's of the same age in different locations may not be of equal size and vice versa.

[15] We will assume that the relationship between albedo and depth is consistent over our region of interest $(20 \mathrm{~km} \times$ $20 \mathrm{~km}$ ) (i.e., that dust co-deposited with the $\mathrm{CO}_{2}$ slab was uniformly distributed). This seems reasonable, as this region is small and mostly flat with no large-scale obstacles to preferentially concentrate atmospheric deposition. Any variations in ice albedo would serve to create a wider spread of observed sizes.

[16] Using the mean diameter of the population $(217 \mathrm{~m})$ and the range of expansion rates quoted above we find the age of this population could range anywhere from $\sim 43$ to 217 Martian years (or 81-408 terrestrial years). Interestingly because the range of sizes is narrow the range of ages is also narrow. The absence of smaller and larger depressions indicates that there was a finite period (that has since ended) where new SCF's on this part of the residual cap

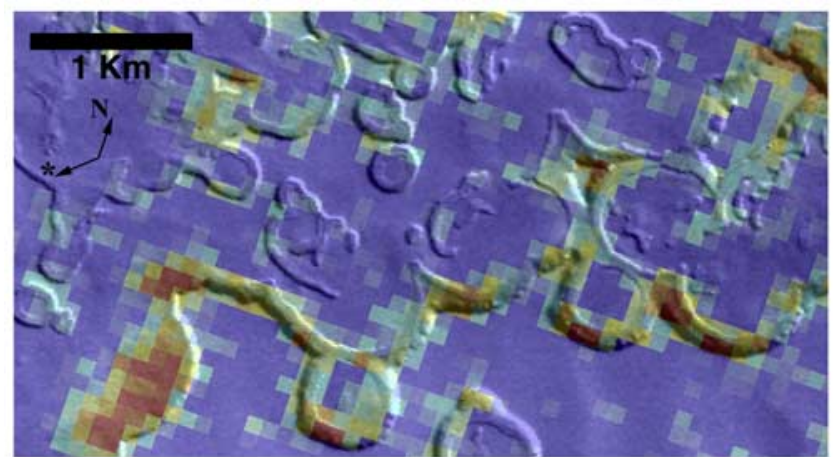

Figure 4. Example of SCF's observed by THEMIS (I01162002) at $86.5^{\circ} \mathrm{S}, 285^{\circ} \mathrm{E}$, and $\mathrm{L}_{\mathrm{s}} 345^{\circ}$. Brightness here is provided by an underlying MOC narrow angle image (E05/02715 at $\mathrm{L}_{\mathrm{s}} 185^{\circ}$ ), while color represents THEMIS temperatures. Direction to sun applies only to MOC image. The reported temperatures range from $\sim 150-160 \mathrm{~K}$ (blue to red). Uncertainties in instrument calibration at such low radiances and atmospheric conditions make the absolute temperatures difficult to retrieve, but temperature differences are reliable. The warmer areas correspond to the floors of the depressions near the walls (moats). These warmer areas, which cannot be $\mathrm{CO}_{2}$ ice, are concentrated around the sun-facing (south) sides of the depressions.

were initiated. We can attempt to estimate the duration of this SCF formation period from the width of this distribution. We can choose the inter-quartile scale $(40 \mathrm{~m}$ in this case) to represent the width of the distribution. This range of sizes represents a spread in starting times of 8 to 40 Martian years (15 to 75 terrestrial years), that again depends on the expansion rate.

[17] The large spreads in the mean population age and length of the formation period are largely due to the large range of possible expansion rates. These uncertainties are correlated though; we can say that the length of time over which these features were forming is about $18 \%$ of the mean age of the population.

[18] Shallow bowls are also found in this location (See Figure 1a) and may be currently forming SCF's. However, no intermediate cases between the shallow bowls and the fully formed flat-floored pits are observed. It may be that we seeing the genesis of another SCF population after an apparent hiatus in new feature formation of at least several decades. However the lack of intermediate cases means that it is difficult to conclusively identify these shallow bowls as SCF precursors.

\section{Relationship to Other Swiss-Cheese Populations}

[19] This population is unique compared to others upon the residual cap in that it is young enough to have large areas of the intervening $8 \mathrm{~m}$ thick layer still undisturbed. In other areas of the residual cap only isolated remnants of an $8 \mathrm{~m}$ thick layer are visible in the process of being eroded by SCF's. Most of these SCF's belong to the second class of features described in Section 2 (i.e., displaying the moats and raised central island evident in Figure 1b). Figure 4 shows one isolated $8 \mathrm{~m}$ thick section being eroded by 
members of this class of SCF. The associated THEMIS temperature data show the moats penetrating to an underlying warmer layer, that because of its elevated temperature cannot be $\mathrm{CO}_{2}$ ice. We interpret this layer to be water ice. This class of SCF was not discussed in paper 1; however these THEMIS data show that they are similar to other SCF's in that a non- $\mathrm{CO}_{2}$ layer also underlies them. Figure 4 shows that the water ice is exposed mostly on the southern parts of the moats, which are the parts that receive the most sunlight. This could be because the southern walls lose $\mathrm{CO}_{2}$ (and so retreat) faster or because the flat floors adjacent to these walls accumulate $\mathrm{CO}_{2}$ more slowly (as these walls are equatorward facing and steeper they reflect more sunlight towards their moats). Reasons behind the central $\mathrm{CO}_{2}$ island are speculated on in Section 6.

[20] Much of the rest of the residual cap appears to be covered in a relatively thinner $\mathrm{CO}_{2}$ layer about $2 \mathrm{~m}$ thick. This thin layer is itself being eroded by SCF's of similar size and smaller than those measured in our region of interest.

\section{Discussion and Speculation}

[21] The narrow distribution of ages for the population of $\mathrm{SCF}$ 's examined in our region of interest indicates that they all began forming close together in time compared with their overall lifetimes. The lack of smaller and larger features in the size distribution indicates that conditions suitable for the initiation of these features existed for some finite period of time, before and after which no new features formed. In periods where no new features are being initiated, existing SCF's can continue to grow. The switching mechanism between periods where new SCF's are being initiated and periods where they are not is uncertain; these timescales are too short to be associated with orbital change and the atmosphere itself has very little memory even on annual timescales [Liu et al., 2003]. One possibility is perhaps the slow redistribution of dust on a regional or even planet wide scale into preferred areas, which changes the albedo pattern with respect to the (by comparison) invariable elevation pattern. Changing the distribution of warm areas on the surface could possibly switch the climate and atmospheric circulation patterns into some other mode leading to differing conditions on the residual cap and a resetting of dust to its original configuration. Averaged over Martian millennia this behavior could be considered to be steady state.

[22] The shallow bowls that are discussed at the end of Section 4 (one of which appears in Figure 1a) may be a new generation of SCF's. If this is the case we would expect from our modeling that these features would be less than 30 Martian years old. One significant event in Martian history that may have been responsible for their genesis is the 1971 global dust storm. This would lead to increased erosion of the residual cap but it is unclear why that would initiate the growth of isolated depressions. If the number density of features is an indication of the severity of the event that initiated them, then the event which initiated the main population discussed in this paper must have been much more severe.

[23] In our modeling we have tuned the albedo of flat surfaces to return to the same elevation at the end of each year. If this condition were relaxed then $\mathrm{CO}_{2}$ ice could acuminate on flat surfaces. $\mathrm{CO}_{2}$ could be effectively redis- tributed from within the SCF's to flat areas elsewhere on the residual cap in this way.

[24] The raised islands and moats within the larger SCF's present other problems. Once water ice is exposed at the surface it becomes very hard to cover it again with enough $\mathrm{CO}_{2}$ to last the whole year without changing the environmental conditions [Jakosky and Haberle, 1990]. One possible explanation is that the larger depressions initially formed in a similar way to the smaller depressions. Some environmental change allowed a few meters of $\mathrm{CO}_{2}$ and dust to settle in their centers. Recent (a few Martian decades to centuries ago) environmental change in the opposite sense could make exposed water ice remain exposed. If the walls were retreating throughout these periods (perhaps at varying rates) then the retreat since the last environmental change would leave a moat of exposed water ice. Alternatively moats may just be a property of large SCF's where the center no longer feels any appreciable radiative effect from the walls. In this picture exposed water ice on flat surfaces would currently be prone to being covered by year-round $\mathrm{CO}_{2}$ but the extra radiation reflected from the walls of the depression could create a small zone where the opposite is true.

[25] Some of these ideas can be tested by further cataloging properties of SCF's throughout the entire residual cap and more detailed modeling of the effects of local control on the $\mathrm{CO}_{2}$ budget.

[26] Acknowledgments. This work was made possible by funding from the Mars data analysis and Mars fundamental research programs. Thanks to the MOLA team and THEMIS (especially Greg Neumann) for such great access to their data and Ken Tanaka and Eric Kolb who provided the outline of the residual cap. Thanks to Norbert Schorghofer for prompting the size measurements and providing comments.

\section{References}

Byrne, S., and A. P. Ingersoll, A Sublimation Model for Martian South Polar Ice Features, Science, 299, 1051-1053, 2003.

Christensen, P. R., B. M. Jakosky, H. H. Kieffer, M. C. Malin, H. Y. McSween, K. Nealson, G. L. Mehall, N. Gorelick, S. H. Silverman, S. Ferry, and M. Caplinger, The Thermal Emission Imaging System (THEMIS) for the Mars 2001 Odyssey Mission, Space Sci. Reviews, submitted, 2003.

Jakosky, B. M., and R. M. Haberle, Year-to-Year Instability of the Mars South Polar Cap, J. Geophys. Res., 95, 1359-1365, 1990.

Kieffer, H. H., Mars South polar spring and summer temperatures: A residual CO2 frost, J. Geophys. Res., 84, 8263-8288, 1979.

Kolb, E. J., K. L. Tanaka, and J. A. Skinner, A new mapping approach for highland materials in the south polar region of Mars (abstract 2105), 34th Lunar Planet. Sci. Conf., Houston, TX, 2003.

Leighton, R. B., and B. C. Murray, Behavior of carbon dioxide and other volatiles on Mars, Science, 153, 136-144, 1966.

Liu, J., M. I. Richardson, and R. J. Wilson, An assessment of the global, seasonal, and interannual spacecraft record of Martian climate in the thermal infrared, J. Geophys. Res., in press, 2003.

Malin, M. C., and K. S. Edgett, MGS MOC: Cruise through primary mission, J. Geophys. Res, 106, 23,429-23,570, 2001.

Malin, M. C., M. A. Caplinger, and S. D. Davis, Observational evidence for an active surface reservoir of solid carbon dioxide on mars, Science, 294, 2146-2148, 2001.

Thomas, P. C., M. C. Malin, K. S. Edgett, M. H. Carr, W. K. Hartmann, A. P. Ingersoll, P. B. James, L. A. Soderblom, J. Veverka, and R. Sullivan, North-south geological differences between the residual polar caps on Mars, Nature, 404, 161-164, 2000.

Thomas, P. C., S. W. Squyres, K. E. Herkenhoff, A. Howard, and B. C. Murray, Polar deposits of Mars, in Mars, edited by H. H. Kieffer, B. M. Jakosky, C. W. Snyder, and M. S. Matthews, Univ. of Arizona Press, Tucson, 767-795, 1992.

S. Byrne and A. P. Ingersoll, Division of Geological and Planetary Sciences, California Institute of Technology, Pasadena, CA 91125, USA. 